LM-03K038

April 24, 2003

\title{
Physical Basis for the Maximum Thermal Radiation Emission between Materials
}

\author{
P.F. Baldasaro and J.F. Beausang
}

This report was prepared as an account of work sponsored by the United States Government. Neither the United States, nor the United States Department of Energy, nor any of their employees, nor any of their contractors, subcontractors, or their employees, makes any warranty, express or implied, or assumes any legal liability or responsibility for the accuracy, completeness or usefulness of any information, apparatus, product or process disclosed, or represents that its use would not infringe privately owned rights. 


\title{
PHYSICAL BASIS FOR THE MAXIMUMI THERMIAL RADIATION EMISSION BETWEEN MATERIALS
}

\author{
PF Baldasaro and JF Beausang* \\ Lockheed Martin, PO Box 1072, Schenectady, NY 12301-1072 \\ *beausjo@kapl.gov \\ 518-395-4046
}

\begin{abstract}
An analytic basis for the limit on intra-media thermal radiation transport has been obtained as a simple function of temperature and material optical properties (n,k). It is shown that optical parameters determine the maximum radiative energy transfer rate by altering media radiative state density and energy density. Quantitative analysis shows that intra-media radiative transfer rates may exceed the radiation into free space as described by the Stephan-Boltzmann equation by several orders of magnitude. The frequency dependence of the optical properties further alters the expected blackbody spectral dependence. This generalized formulation of the limit to thermal radiation transfer in terms of media optical properties expands the understanding and future potential of radiative processes.
\end{abstract}




\section{Introduction}

Thermal radiation transfer limits are often viewed in terms of the Stephan-Boltzmann relationship:

$$
Q=\sigma T^{4}
$$

Where: $\quad Q$ = Surface radiative heat flux

$\sigma \quad=$ Stephan-Boltzmann constant

$\mathrm{T}=$ Absolute surface temperature

The Stephan-Boltzmann relationship (1) is based on a free space equilibrium energy density argument, which necessarily presents radiation transfer as limited by free space parameters, independent of radiator material properties. However, there is abundant literature ${ }^{1-7}$ to demonstrate that radiation transfer between materials does not strictly obey (1), and that intra-media properties greatly affect the radiation transfer rate. The existing literature describes radiative energy transfer as an extension of classical electromagnetic theory by considering time-averaged electromagnetic fields that arise from thermally generated fluctuating electrical currents within the media. The nature and limit of intramedia radiative energy is not clear from the electromagnetic approach presented in the literature, and the purpose of this paper is to provide an alternative physical basis, and clear analytical limit for thermal radiation transfer.

\section{Analysis}

For analysis purposes, it is convenient to expand upon the original formulation used by Plank in the derivation of Blackbody radiation ${ }^{8}$ :

1) the consideration of an equilibrium cavity having thermal radiation energy density, $\mathrm{U}$, which is determined as the product of radiation state density, $\mathrm{D}$, and equilibrium occupation number, $\mathrm{N}$, so that $\mathrm{U}=\mathrm{D} \cdot \mathrm{N}$, and

2) the determination of net thermal radiation energy flux, $Q$, as the product of energy density times an effective radiation velocity, $V$, so that $Q=U \cdot V$.

Three distinct intra-media radiation transfer situations are considered:

1) between an electronic medium and free space (no charge)

2) between an electronic medium and a dielectric medium (no free charge)

3) between an electronic medium and an electronic medium (free charge). 


\section{Case 1: Radiation transfer between an electronic medium and free space}

The transfer of thermal radiation into free space can be viewed as the direct contact between an electronic medium (radiator) and free space, as shown in Fig. la and considered by Planck in his original Blackbody formulation. Analysis begins with consideration of the spectral density, $D(\omega)$, of photons in free space ${ }^{9}$ :

$$
D(\omega)=2 \cdot 4 \pi \cdot\left(\frac{1}{\lambda^{2}}\right) \cdot d\left(\frac{1}{\lambda}\right)=\frac{\omega^{2}}{\pi^{2} c^{3}} d \omega
$$

The factor of 2 indicates the photon polarization and $4 \pi$ is the total solid angle. The number of photons, $N(\omega)$, in a given radiation state of energy $\hbar \omega$ is:

$$
N(\omega)=\left(\exp \left(\frac{\hbar \omega}{k T}\right)-1\right)^{-1}
$$

The thermal radiation energy density, $u(\omega)$, in a free space (FS) cavity can then be calculated:

$$
u_{F S}(\omega)=D(\omega) \cdot N(\omega) \cdot \hbar \omega=\frac{\hbar \omega^{3}}{\pi^{2} c^{3}} \cdot\left(\exp \left(\frac{\hbar \omega}{k T}\right)-1\right)^{-1} d \omega
$$

And the total thermal energy integrated over the entire spectrum in the cavity is:

$$
U_{F S}=\int_{0}^{\infty} u_{F S}(\omega) d \omega=\frac{4}{c} \cdot \frac{\pi^{2} k^{4}}{60 c^{3} \hbar^{3}} T^{4}=\frac{4}{c} \sigma T^{4}
$$

where: $\quad \sigma=$ Stephan-Boltzmann constant

$\mathrm{k}=$ Boltzmann constant

$\mathrm{c}=$ speed of light in vacuum

$\mathrm{T}=$ cavity temperature

The effective velocity $V_{\mathrm{FS}}$ of the thermal radiation crossing an arbitrary plane in the cavity is obtained by averaging over the solid angle, $\Omega$ :

$$
V_{F S}=\int c \cos \theta \frac{d \Omega}{4 \pi}=\int_{0}^{\frac{\pi}{2}} c \cos \theta \frac{2 \pi \sin \theta}{4 \pi} d \theta=\frac{c}{4}
$$

The product of $V_{F S}$ and $U_{F S}$ yields the net thermal radiation flux, $Q_{F S}$, across a plane in the cavity, which is the familiar Blackbody formulation for thermal radiation flux into vacuum: 


$$
Q_{F S}=U_{F S} \cdot V_{F S}=\sigma T^{4}
$$

In this context, radiation transfer is limited by the radiation energy density of free space, not the photonic energy density of the radiating body.

It is instructive to consider the components of (7) at a given temperature. For example, at $1000^{\circ} \mathrm{C}$ the net Blackbody thermal flux is $\sim 20 \mathrm{~W} / \mathrm{cm}^{2}$, which is the product of an internal energy $U$ of $\sim 2 \times 10^{-9} \mathrm{~J} / \mathrm{cm}^{3}$ and an effective radiation velocity $V_{\text {eff }}$ of $\sim 10^{10} \mathrm{~cm} / \mathrm{sec}$. This analysis illustrates blackbody radiative energy transfer as low energy density moving at high speed. Next, a generalized version of this approach is applied to explain the additional radiative heat transfer when the receiving medium contains bound and mobile charges.

\section{Case 2: Radiation transfer between an electronic and dielectric media}

Increased radiative heat transfer from an electronic radiator occurs when vacuum is replaced by a higher index of refraction, $n(\omega)$, dielectric (DI) medium and can be understood from a similar Planckian formulation as in Case 1. The increased index of the receiving dielectric media is a measure of the greater photon state density $D(\omega)$ compared to free space (Fig. 1b) since the wavelength of the radiation is reduced, but the frequency is unchanged:

$$
\lambda_{\mathrm{DI}}=\lambda_{\mathrm{FS}} / \mathrm{n}
$$

The reduced wavelength of the dielectric medium leads to an increased concentration of radiation states $\left(1 / \lambda^{3}=\mathrm{n}^{3} 1 / \lambda_{\mathrm{FS}}{ }^{3}\right)$, and a corresponding $\mathrm{n}^{3}$ increase in thermal radiation energy density over that of free space:

$$
u_{D I}(\omega)=n(\omega)^{3} u_{F S}(\omega)
$$

The dielectric medium holds $\mathrm{n}^{3}$ more waves, and thus $\mathrm{n}^{3}$ more internal energy. The effective dielectric velocity, $\mathrm{V}_{\mathrm{Dl}}$, however, is reduced relative to the free space value by the factor of $1 / \mathrm{n},\left(\mathrm{V}_{\mathrm{DI}}=\mathrm{V}_{\mathrm{FS}} / \mathrm{n}\right)$ to yield a net radiation transfer rate:

$$
Q_{D I}=\bar{n}^{2} Q_{F S}=\bar{n}^{2} \sigma T^{4}
$$

Where $\vec{n}$ is the frequency-weighted refractive index, and is defined for an arbitrary parameter $x$ as:

$$
\bar{x}=\frac{\int_{0}^{\infty} x(\omega) \cdot \omega^{3} \cdot\left[\exp \left(\frac{\hbar \omega}{k T}\right)-1\right]^{-1} d \omega}{\int_{0}^{\infty} \omega^{3} \cdot\left[\exp \left(\frac{\hbar \omega}{k T}\right)-1\right]^{-1} d \omega}
$$


Equivalently, (10) can be represented in terms of material dielectric properties, using the classical relationships between optical and dielectric properties:

$$
\varepsilon=\left(\varepsilon^{\prime}+i \varepsilon^{\prime \prime}\right) \varepsilon_{0}
$$

Where,

$$
\begin{aligned}
& \varepsilon^{\prime}=n^{2}-k^{2} \\
& \varepsilon^{\prime \prime}=2 n k
\end{aligned}
$$

Since $\mathrm{k}=0$ in the dielectric, an equivalent form of (10) in terms of dielectric properties can be expressed:

$$
Q_{D I}=\bar{\varepsilon}^{\prime} Q_{F S}=\bar{\varepsilon}^{\prime} \sigma T^{4}
$$

Equation (13) shows that the magnitude and frequency dispersion of radiation within a dielectric medium deviates from the classical free space form by the material dependent functionality of $\varepsilon^{\prime}(\omega)$. The same material radiating a heat flux $Q_{F S}$ into vacuum will radiate $n^{2} Q_{F S}$ into a dielectric of refractive index $n(\omega)$. The photonic energy is always present in the radiator, but the transfer rate is limited by the energy storage capability $\left(\varepsilon^{\prime}\right)$ of the receiving medium.

\section{Case 3: Radiation transfer between electronic media}

The magnitude of radiation transfer between media with mobile electrons (EL) can also be treated via a Planckian approach with some conceptual alteration to account for electron screening effects. Classical electromagnetic theory ${ }^{4-7}$ shows that propagating modes of radiation cannot exist within an electronic material because of electron screening. However, the screening process does not extinguish the existence of radiative states (Fig. 1c), but rather serves as an interaction mechanism that impedes the effective radiative velocity.

The radiative velocity within the medium can be calculated as the product of frequency and an effective wavelength $\lambda_{\mathrm{EL}}$ in the electronic medium that is reduced relative to the free space value by electron screening. For good conductors $\left(\sigma_{0} \gg \omega \varepsilon^{\prime \prime} \varepsilon_{0}\right.$, or equivalently, $\mathrm{k}>1$ ), the effective wavelength can be approximated as the skin depth, $\delta^{10}$ :

$$
\lambda_{E L} \approx \delta=\frac{1}{\sqrt{\frac{\omega}{2} \mu \sigma_{0}}}
$$

Where: $\quad \mu=$ magnetic permeability $=\mu_{0}$

$\sigma_{0}=$ electrical conductivity $=2 \mathrm{nk} \omega \varepsilon_{0}$, for good conductors 
Using the optical definition for conductivity results in a simplified relationship for (14) that is similar to (8) and illustrates the role of the extinction coefficient in determining the effective radiative wavelength:

$$
\lambda_{E L}=\frac{c}{\omega \sqrt{n k}}=\frac{\lambda}{\sqrt{n k}}
$$

The effective velocity in electronic media is then:

$$
V_{E L} \approx \omega \cdot \lambda_{E L}=\frac{c}{\sqrt{n k}}
$$

Following the same rationale of cases 1 and 2, the energy density increases by the cube of the effective wavelength, $(\mathrm{nk})^{3 / 2}$, but the effective velocity decreases by $(\mathrm{nk})^{1 / 2}$, resulting in a radiative energy flux that is a factor of (nk) higher than the free space limit:

$$
\begin{aligned}
U_{E L}(\omega) & =[n(\omega) k(\omega)]^{3 / 2} U_{F S} \\
Q_{E L} & =\bar{n} \bar{k} \cdot Q_{F S}=\bar{n} \bar{k} \cdot \sigma T^{4}
\end{aligned}
$$

Alternatively, (18) can be reformulated in terms of electronic dielectric properties:

$$
Q_{E L}=\frac{\bar{\varepsilon}^{\prime \prime}}{2} Q_{F S}=\frac{\bar{\varepsilon}^{\prime \prime}}{2} \cdot \sigma T^{4}
$$

Equation (18) shows that the magnitude and frequency dispersion of radiative energy within an electronic medium is determined by the material dependent functionality of $\varepsilon^{\prime \prime}(\omega)$.

\section{Discussion}

Equations (13) and (19) in Section II show that the dielectric frequency functionality of $\varepsilon^{\prime}$ and $\varepsilon^{\prime \prime}$ determines the magnitude and spectral characteristics of radiative energy transfer between materials. For example, Fig. 2 shows the frequency dependent refractive index for silicon in the infrared range where the refractive index exceeds 3.5 or correspondingly, $\varepsilon^{\prime}$ exceeds 10 . Therefore, the radiative energy transfer between silicon media may be more than an order of magnitude greater than into free space, depending on the frequency. Reference 11 provides experimental evidence of the enhanced radiative transfer between dielectric materials.

For electronic materials, the potential increase in radiative heat flux is more dramatic. Figure 3 shows that the measured values of $n, k$ for a good conductor such as gold in the infrared range exceeds 30 , supporting a several order of magnitude increase in radiative 
energy transfer relative to free space, again depending on frequency. Reference 10 provides experimental evidence of enhanced radiative transfer between electronic media.

It is equally interesting to consider radiative spectral characteristics of intra-media energy transfer. As shown in Figures 2 and 3, optical and dielectric parameters have a strong frequency dependence (several orders of magnitude), which makes the knowledge and selection of materials critical for applications utilizing the radiative energy.

Preferential use of only the increased radiative energy within a dielectric or electronic media without simultaneously accessing the conductive phonon energy is not trivial because the source of the increased radiative energy is the internal charges of the medium. This difficulty does not apply to the limited state density of free space, as radiation will propagate to infinity. Therefore, the increased transfer of thermal radiative energy between media must occur via intimate contact, or very close spacing between the media as analyzed in References 2-4. Efficient radiation transport between media requires spacing separations less than the wavelength of the radiation of interest, typically less than a micron depending on wavelength of interest $(\lambda / n)$. This problem is exacerbated in the case of electronic media, where screened wavelengths may be even smaller $\left(\lambda / V_{n k}\right)$. The utilization of the enhanced radiative energy within media represents a new technological challenge in terms of dimensional control in the presence of a temperature gradient.

In practical configurations the precise magnitude of the energy transfer depends on temperature, spacing, angle, and emissivity and may require evaluation of the integral solutions found in Reference 5. The purpose of this paper is to provide a limit on the radiative energy transfer that can be used as a rule-of-thumb for selecting optimal materials as well as to provide a simpler physical model by which to understand the enhanced radiative transfer process.

\section{Summary}

The presence of charge within materials greatly increases the radiative energy transfer capability relative to free space. Dielectric media offer an $\varepsilon^{\prime}=n^{2}$ increase, (roughly an order of magnitude ) in radiative transfer rate, while electronic media offer an $\varepsilon^{\prime \prime} / 2=n k$ increase over traditional free space Blackbody transfer of $\sigma T^{4}$. The increased radiative energy density presents new opportunities for radiative energy utilization, at the cost of dimensional control at the sub-micron level. 


\section{References}

1 R. S. DiMatteo, et. al., Applied Physics Letters. 79, 1894 (2001).

2 M. D. Whale, Doctoral Thesis. Massachusetts Institute of Technology (June 1997) (unpublished).

3 JE Raynolds, AIP Conference Proceedings. 460, 49 (1999).

4 J.L. Pan, H.K.H. Choy, and C.G. Fonstad, IEEE Trans. Electron. Devices., 47, 241 (2000).

5 D. Polder and M. Van Hove, Phys. Rev. B 4, 3303 (1971).

6 C. M. Hargreaves, Philips Res. Rep., Suppl. 5, 1 (1973).

7 M. L. Levin, VG Polevoi, and SM Rytov, Sov Phys. JETP, 6, 1054 (1980).

8 C. Kittel, Thermal Physics, $2^{\text {nd }}$ edition, (1980).

9 R. K. Pathria, Statistical Mechanics. Butterworth Heinemann: Woburn, 1996.

10 C. A. Balanis, Advanced Engineering Electromagnetics, John Wiley and Sons: New York, (1989).

11 P. F. Baldasaro and P Fourspring, STAIF Conference Feb 6, (2003).

12 Electronic Handbook of Optical Constants of Solids, Eds E.D. Palik and Gorachand Ghosh, Academic Press: San Diego, (1999). 


\section{List of Captions}

1. Radiative energy transfer into (a) free space, $n_{F S}=1$ and $k_{F S}=0$; (b) dielectric media, $n_{D P}>1$ and $k D I=0 ;(c)$ electronic media $n_{E L} \neq 1$ and $k_{E L}>0$.

2. Optical properties of silicon

3. Optical properties of gold 


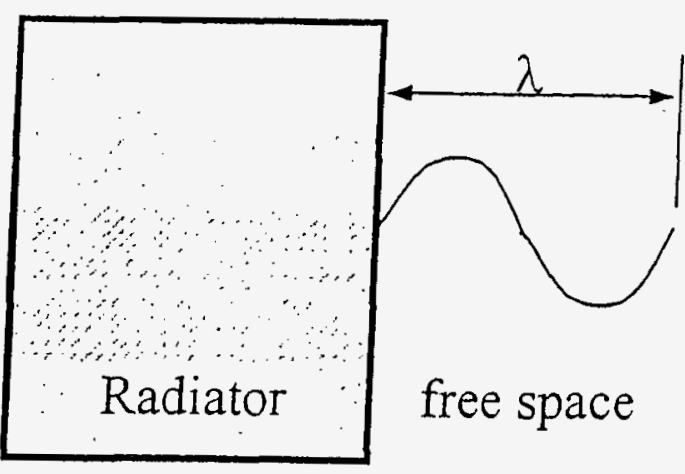

(a)

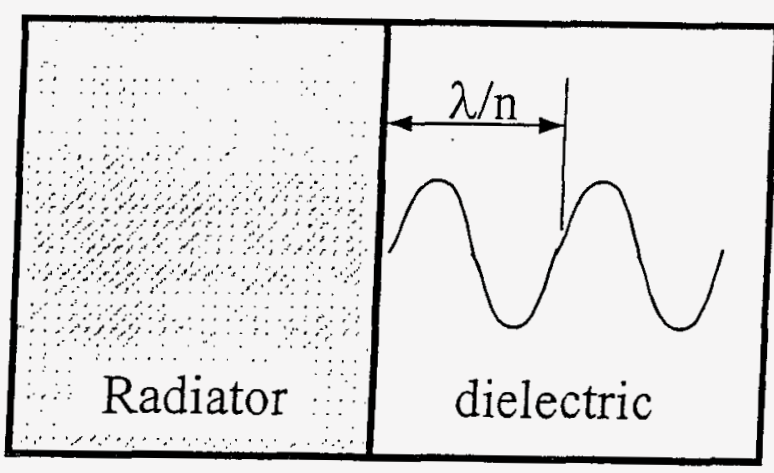

(b)

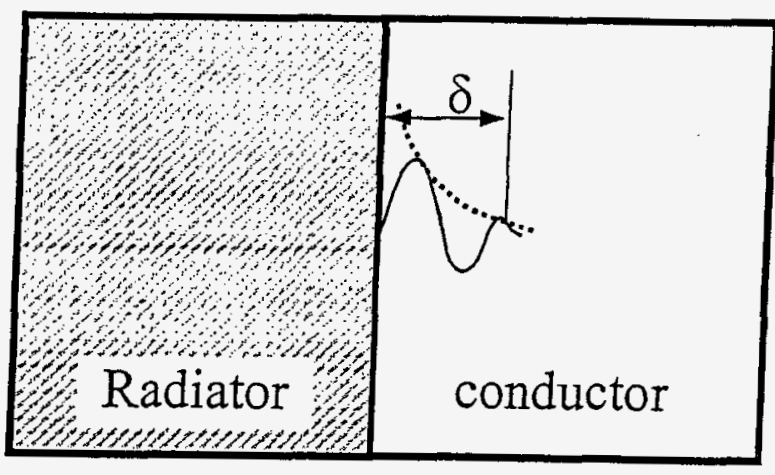

(c) 


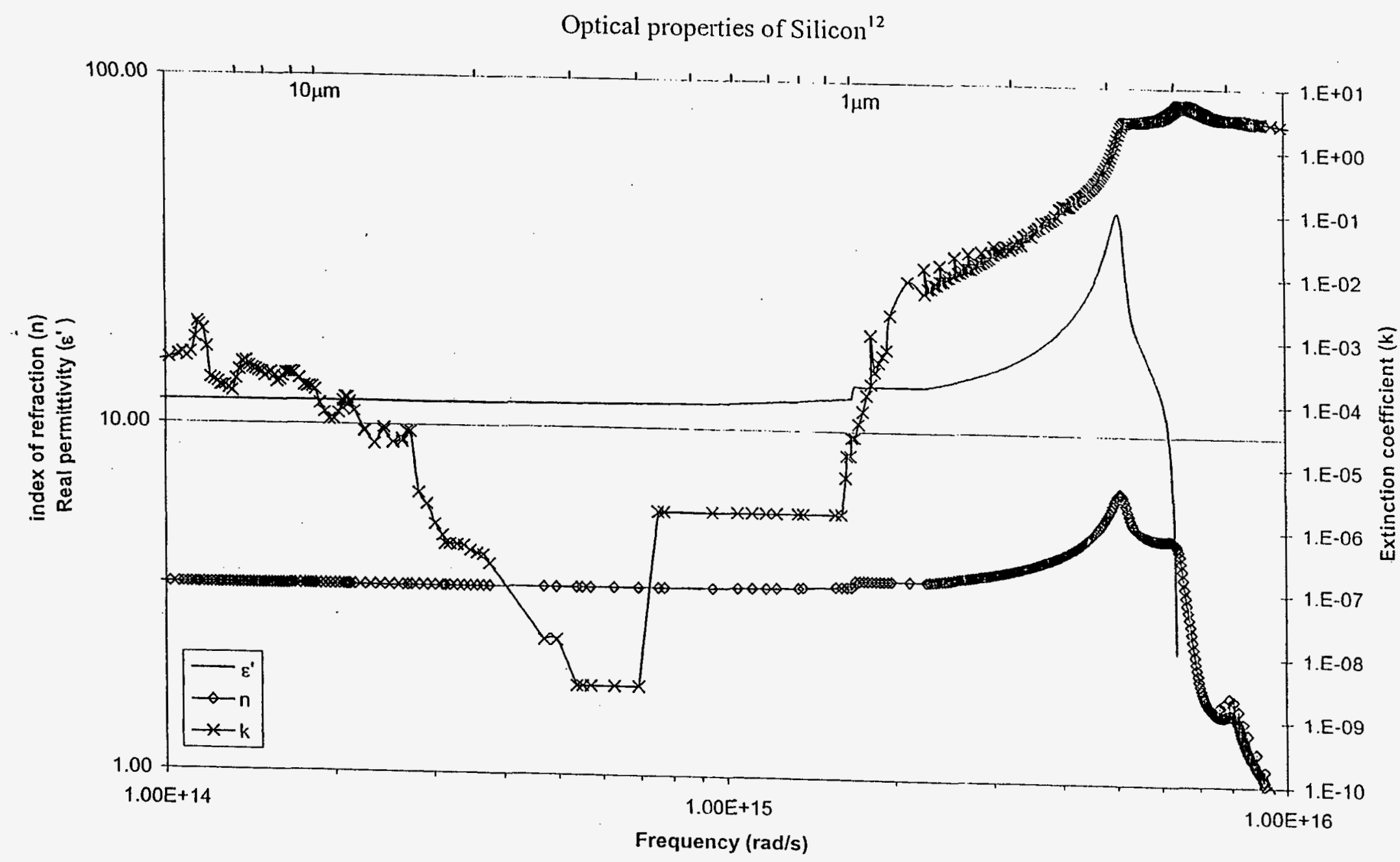




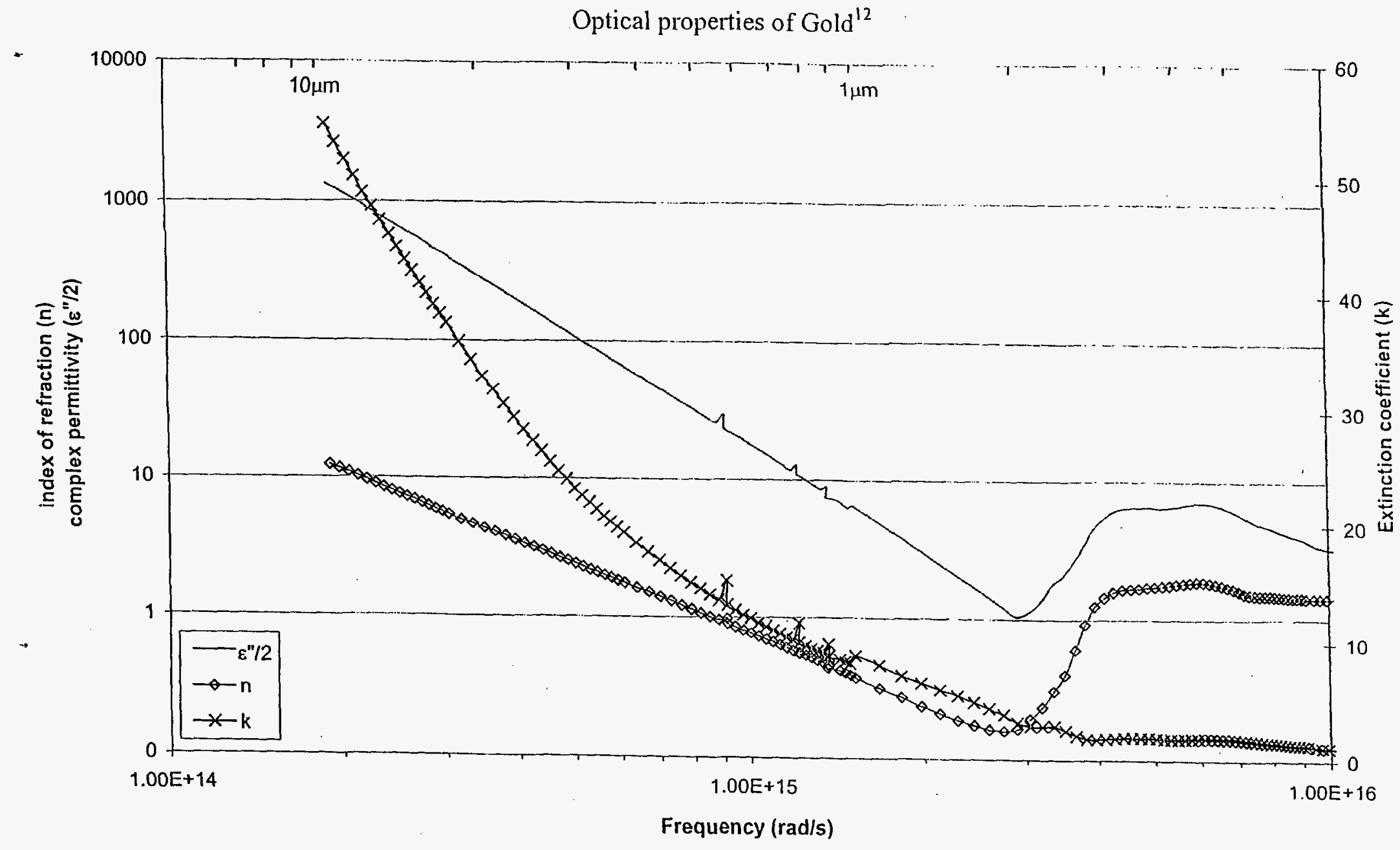

